\title{
Simulation of Molecular Evolution Using Population Dynamics Modelling
}

\author{
Sergei V. Semovski ${ }^{1}$ \\ Limnological Institute SB RAS, Ulanbatorskaya 3, Irkutsk 664033 Russia \\ semovsky@lin.irk.ru
}

\begin{abstract}
Neutral evolution of nucleotide sequences is simulated in the framework of adaptive population dynamics. Simulated speciation events, changes in demographic history, and migration are traced using resulting molecular data. Various scenarios are considered including oneand two-dimensional populations, and competition lottery system.
\end{abstract}

\section{Introduction}

It has been shown recently that DNA sequences retain much more information on the past events than simply the degree of relation between species. The main successes in the direction of interpreting this information have been achieved in the framework of the coalescence theory which has allowed scientists to obtain the demographic history of human beings. We use mathematical modelling to simulate evolutionary and demographic processes in populations consisting of objects with different ecological parameters such as mobility, number of progeny etc., and include into each object tracer mutable "DNA sequences", which are extracted after each simulation and analysed with various molecular phylogenetic approaches. This will enable us to match the inferences to the known history.

Population dynamics is a flexible tool, giving opportunities to investigate various practically important ecological situations. In the Lab of molecular systematics, at the Limnological Institute we concentrate mainly on studies of benthic invertebrates (see [1])). Several models of molecular evolution valid for low-mobile species are presented here including a one-dimensional population, which is the model of littoral of an ancient lake, and different ecological and evolutionary patterns in a two-dimensional community.

\section{Model}

Let us consider a population existing in the environment with limited resource, according to a generalisation [2] of the well-known logistic equation:

$$
\frac{d S(x, t)}{d t}=r \cdot S(x, t) \cdot\left[1-\int \frac{C(x-y) S(y, t)}{K(x)} d y\right] .
$$


Here $S(x, t)$ denote the number of individuals with value of ecological character (polygen) $x$ in time $t$. Resource distribution and competition between individuals are considered to be gaussian functions:

$$
K(x)=K_{0} \exp \left[-\frac{\left(x-x_{0}\right)^{2}}{2 \sigma_{K}^{2}}\right]
$$

and

$$
C(z)=\exp \left[-\frac{z^{2}}{2 \sigma_{C}^{2}}\right] .
$$

The probability of progeny survival depends on the genetic distance between the parents. The local minimum in zero point is due to inbreeding, and the local maximum is due to heterosis. Hybrid depression at genetic distances larger then $K_{0}$ causes reproductive isolation of genetically different organisms.

Rare mutations occur with the probability equal to 0.01 and their deviation from parent genotype corresponds to gaussian distribution

$$
\Psi(x)=\left(2 \pi \sigma_{\Psi}^{2}\right)^{-1} \exp \left[-x^{2} / \sigma_{\Psi}^{2}\right],
$$

where $\sigma_{\Psi}^{2}$ is dispersion of the genotype of the rare mutant. The individualoriented model has been used for all calculations.

For spatially distributed individuals the generalization of the model [2] is proposed in [3. The general idea of application of (11) is in the introduction of the Gaussian competition between individuals in the space. Then integral operator on the right side of (1) becomes multidimensional and contains an additional kernel $C_{s p}(z)=\exp \left[-\frac{z^{2}}{2 \sigma_{s p}^{2}}\right]$ responsible for competition in the space. Here $z$ is the distance between individuals in the space and $\sigma_{s p}$ defines the intensity of spatial competition. Note that for the sake of calculation time it is possible to use rectangular kernel instead of Gaussian. Such formulation will correspond to the limited capacity of the spatial cell. It is possible to use the same attempt to describe the dynamics of interacting populations. If competition for the same resource takes place in the space, individuals from different populations interact according to the integral operator with kernel $C_{s p}$, however, in the "genetic" space of the quantative trait competition is intra-specific.

In order to bring the neutrally evolving marker into the individually oriented model we consider that each individual bears a neutrally evolving nucleotide sequence represented here by integer vector with elements having values from 1 to 4 We consider all mutations in this sequence neutral that have no impact on the adaptation of individuals. The progeny inherits parental sequence with mutations occurring with the probability of .001 per generation. In the case of a bisexual population this sequence would be considered to be inherited from one of the parents only (maternal inheritance), which corresponds to mitochondrial DNA. The set of neutral sequences resulting from a simulation was used for phylogenetic inferences, which were performed with the program fitch from the package PHYLIP [5]. The phylogenetic trees were visualized with the program njplot [4. Program system IDL (Reseach Systems Inc.) has been used intensively for computations and results presentation. 


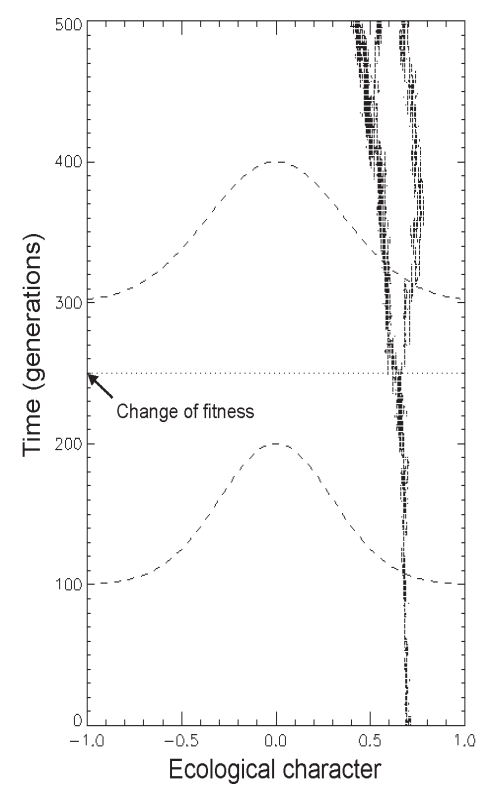

Fig. 1. Simulation of evolutionary history using model (1)

\subsection{Molecular Evolution}

Incorporating the molecular evolution in the population dynamics model (1) produces a suitable tool to validate those methods of bioinformatics which have been developed to reconstruct details of evolutionary history based on the present samples of those DNA sequences which are selectively neutral. One of important questions is the correlation of demographic changes in population history and speciation events with past changes in the environment. In [6] some details are presented of the application of the model (I) to reconstruct the evolutionary history for different scenarios of environmental change impact on speciation intensity. In Fig. 1 one can see formation of genetically isolated subpopulations after change in fitness. Fig. 2 demonstrates application of molecular phylogeny to resulting molecular data.

\subsection{Spatial Patterns of Speciation}

Bottom communities of ancient lakes demonstrate various spatial patterns in speciation. In the shallow littoral zone along the shore the benthic community presents approximately a one-dimensional population. In 3] using a generalization of the model (1) different situations of speciation in space have been demonstrated depending on mobility of organisms. For low-mobile species formation of different, genetically isolated colonies is possible, which is close to allopatric speciation but without geographical barriers. For moderate mobility parapatric 


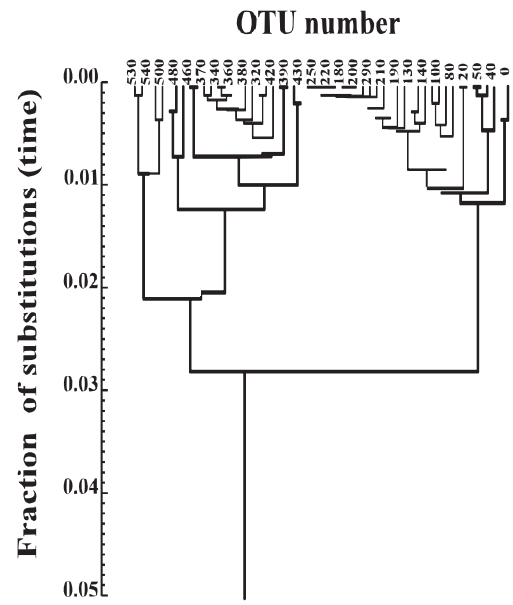

Fig. 2. Reconstruction of evolutionary history of the case presented in Fig. I using resulting molecular data

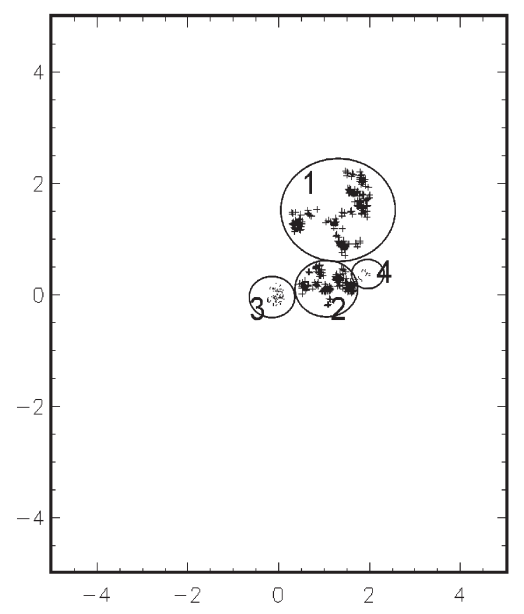

Fig. 3. Speciation patterns for two competitive populations on a plane. 1 and 2 are spatial positions of two genetically different morphs of population $A, 3$ and 4 are spatial positions of two genetically different morphs of population $B$

speciation takes place, for which zones of genetic contact are typical, both permanent and temporary, with migration or without it. For highly mobile organisms situation becomes close to zero-dimensional case (11) and we have sympatric speciation with full mixing of newly formed subpopulations. 


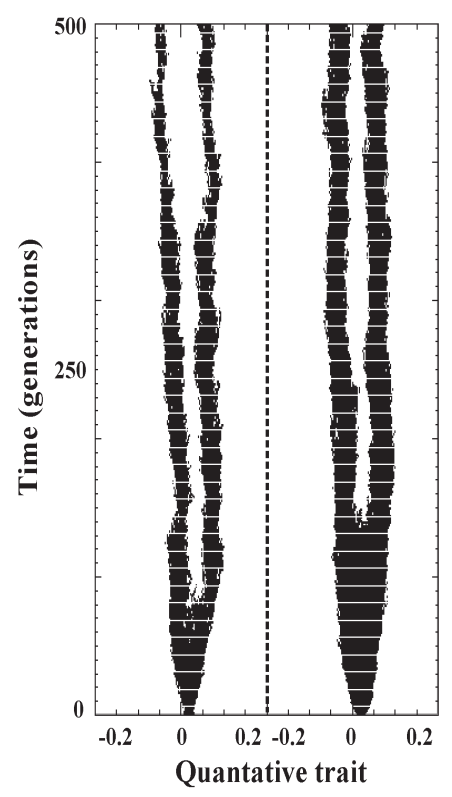

Fig. 4. Co-evolution patterns of two competing populations on a plane

\subsection{Co-evolution in Competing Populations}

Co-evolution and co-speciation is known for many forms of interaction between species in nature. It is possible for host and parasite pairs, preys and predators. We have a plan to investigate co-evolutionary patterns for different forms of ecological interaction. Results presented here are derived using a model of coevolution of spatially distributed organisms which compete for a limited resource.

For many natural communities the well-known principle of competitive exclusion is not valid. One of the examples one can find in benthic ecosystems. One of verbal models is based on the idea of "competition lottery", when newborn offspring have equal probability to occupy areas which are free due to organisms mortality. The numerical realization of this idea is based on a three-dimensional generalization of the model (1D), where one dimension is a quantative trait (polygenus) and two domensions are spatial. Fig. 3. demonstrates spatial patterns of new parapatric (being in contact) genetically diverging morphs for two competitive species on a plane. To neglect boundary effects in calculations we use torus. Co-evolution patterns in the "genetic" space are presented in Fig. 目.

Changes in the environment during evolutionary history can have different consequences for competing populations, correlative and anti-correlative. Fig. 5 demonstrates the analysis of evolutionary history using resulting molecular data and frequency diagram of pair-wise genetic distances of organisms in the population. Peaks in this diagram correspond to moments of population growth[7, or to moments of speciation [6], minima - to population decline or to decrease in 
speciation rate. In Fig. 5 two populations demonstrate opposite reactions to the changes in the model environment. Note that a similar anti-correlation has been noted recently for sand-dwelling and rock-dwelling cichlids in lake Tanganyika [8] using a statistical analysis based on a big array of molecular data.

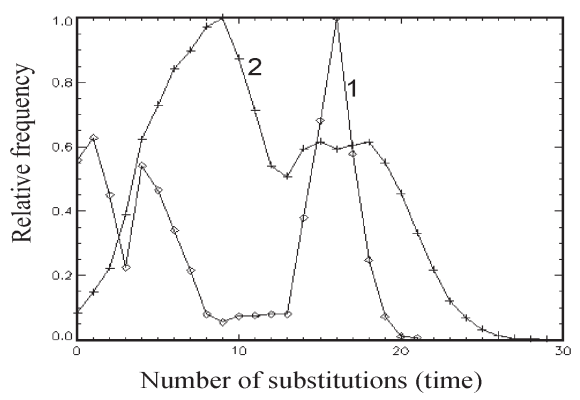

Fig. 5. Frequency diagram for genetic distance (number of substitutions) in two competing populations on a plane which react in different ways to changes in the model environment

\section{References}

1. Sherbakov D.Yu.: Molecular phylogenetic studies on the origin of biodiversity in Lake Baikal. Trends in Ecol.and Evolution 14 (1999) 92-95.

2. Dieckmann U., Doebeli M.: On the origin of species by sympatric speciation, Nature, 400 (1999) 354-357.

3. Semovski S.V., Bukin Yu.S., Sherbakov D.Yu.,: Speciation in one-dimensional population. Int.J.of Modern Phys. 14 (2004), (to appear).

4. Gouy, M. : NJPLOT(m.gouy@biomserv.univ-lyon1.fr)

5. Felsenstein, J.: PHYLIP, Phylogeny Inference Package http://evolution.genetics.washington.edu/phylip.html

6. Semovski S.V., Verheyen E., Sherbakov D.Yu.,: Simulating the evolution of neutrally evolving sequences in a population under environmental changes. Ecol.Modelling, (to appear)

7. Rogers, A.R., Harpending, H. : Population growth makes waves in the distribution of pairwise genetic differences, Mol.Biol.Evol., 9 (1992) 552-569.

8. Nevado B., Sherbakov D.Yu., Verheyen E., : private communications. 